\title{
Heartbeat: socioeconomic deprivation is associated with non-cardiovascular mortality in chronic heart failure patients
}

doi:10.1136/heartjnl-2018-313460

Healthcare costs for patients with chronic heart failure (CHF) account for $2 \%$ of the UK National Health Service budget so that efforts to improve outcomes and reduce hospitalisations in this patient group is relevant to healthcare policy. However, the reasons for recurrent hospitalisation in CHF patients are not well understood. In order to assess whether socioeconomic deprivation, measured by the UK Index of Multiple Deprivation (IMD), contributes to adverse outcomes in CHF patients, Witte and colleagues ${ }^{1}$ analysed data from a prospective cohort of 1802 CHF patients (ejection fraction $45 \%$ or less) followed for a mean of 4 years. The IMD score was associated with non-cardiovascular mortality $(9 \%$ higher risk per 10-unit increase in IMD score; $95 \%$ CI $3 \%$ to $16 \% ; \mathrm{p}=0.003)$, but not cardiovascular mortality (figure 1). Similarly, IMD score was associated with a higher rate of hospitalisation for non-cardiovascular causes. At first glance, these results seem counter-intuitive. However, this data makes evident the need to treat the entire patient with CHF and not just focus on heart failure treatment alone.

In the accompanying editorial, Squire ${ }^{2}$ reminds us that "the societal burden associated with CHF is enormous and growing. Similarly, the individual patient burden associated with a diagnosis of CHF is potentially high; this remains a condition associated with significant morbidity and mortality." He concludes that "It appears clear that patients in areas of high deprivation continue to be at risk of adverse outcomes and that such outcomes may not relate directly to one major diagnosis, such as CHF."

Despite advances in cardiac surgery over the past several decades, atrial fibrillation (AF) continues to occur commonly in the postoperative period. This is an important problem because postoperative $\mathrm{AF}$ is associated with an increased risk of stroke, hospital length

Correspondence to Professor Catherine M Otto, Division of Cardiology, University of Washington, Seattle, WA 98195, USA; cmotto@uw.edu
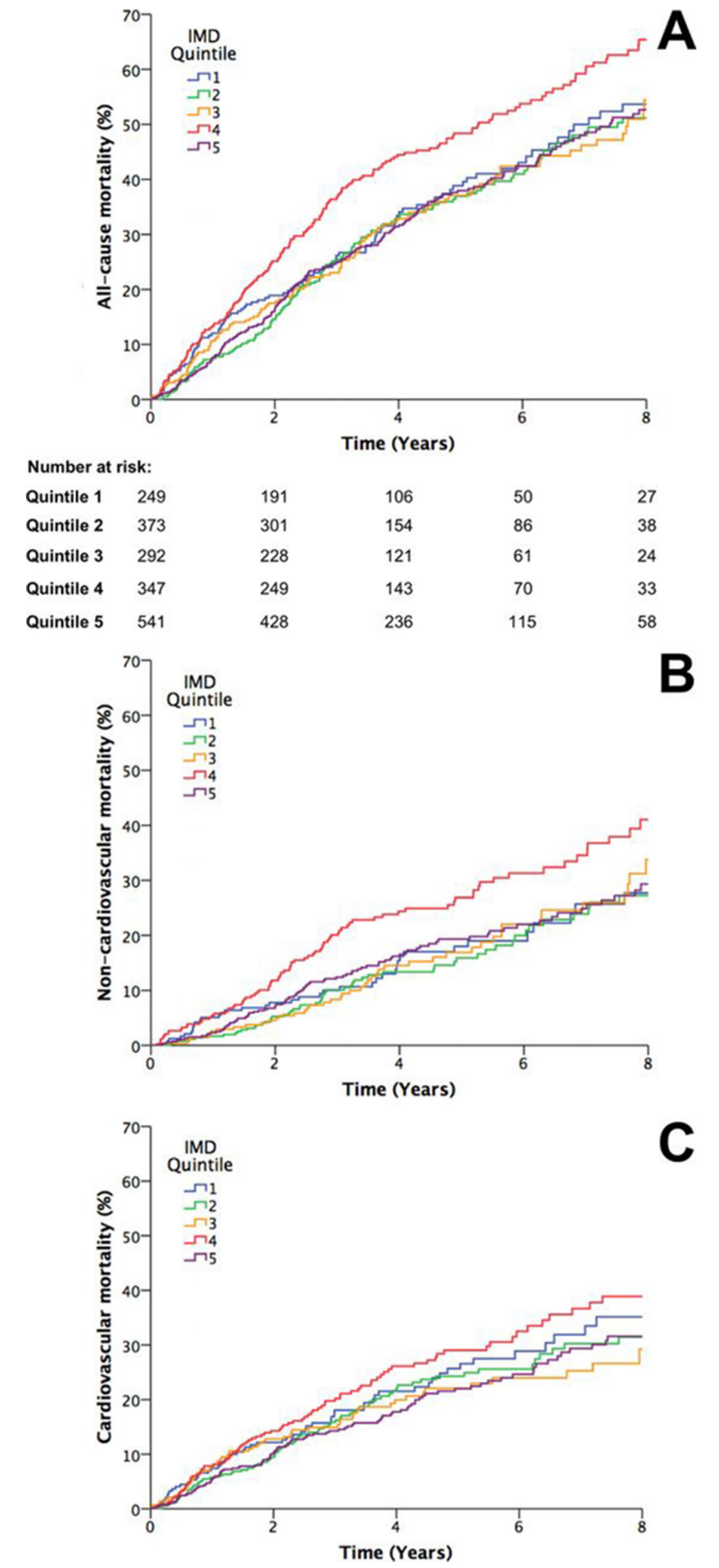

Figure 1 All-cause and mode-specific mortality. (A) Kaplan-Meier curves illustrating all-cause mortality according to Index of Multiple Deprivation (IMD) quintiles ( $p<0.001$ by log-rank test), with numbers at risk indicated below the $x$-axis. (B) Kaplan-Meier curves illustrating noncardiovascular mortality according to IMD quintiles ( $p=0.002$ by log-rank test). (C) Kaplan-Meier curves illustrating cardiovascular mortality according to IMD quintiles ( $\mathrm{p}=0.11$ by log-rank test). 


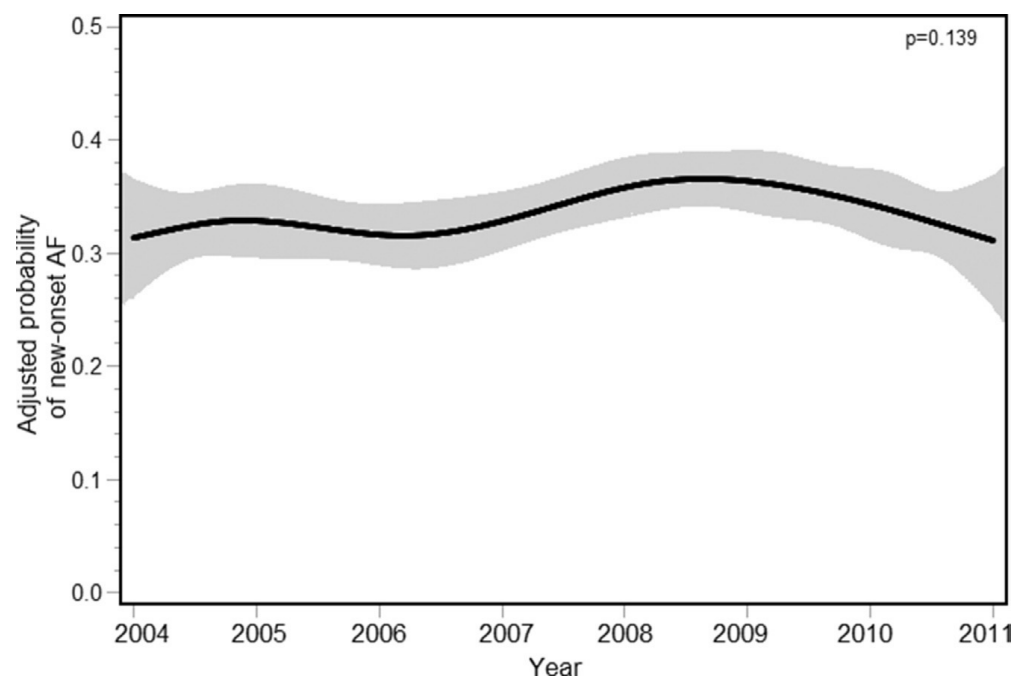

Figure 2 Adjusted probability of new-onset post-coronary artery bypass graft surgery (CABG) over time. Society of Thoracic Surgeons risk-adjusted time trend and corresponding $p$ value showing the probability of new-onset post-CABG atrial fibrillation (AF) over the study period. The thick black line represents the point estimate, with the shaded grey area representing the $95 \% \mathrm{Cl}$.

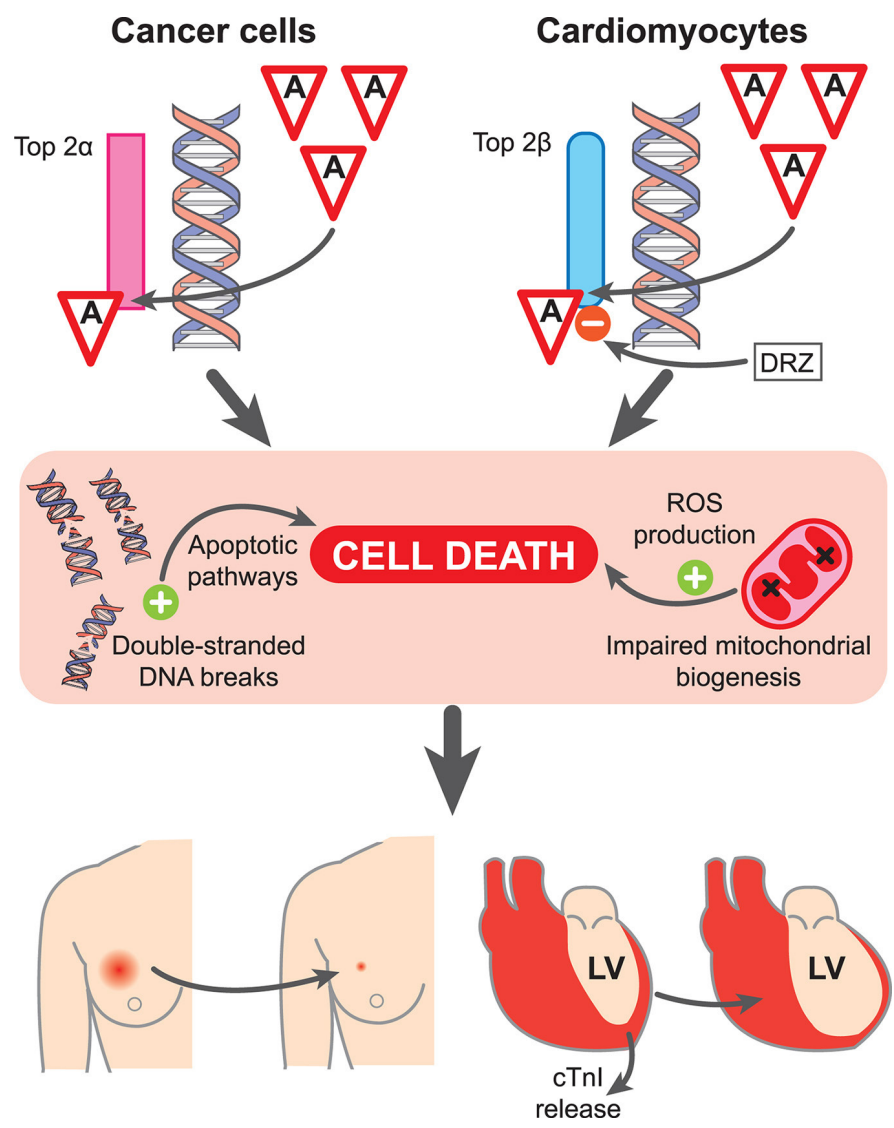

Figure 3 Mechanism of anthracycline-mediated cell injury and death in cancer cells and cardiomyocytes. The therapeutic effect of anthracyclines against cancer cells is mediated through inhibition of Top $2 \alpha$. In cardiomyocytes, Top $2 \beta$ is inhibited. Top 2 inhibition in both cell types causes accumulation of double-stranded DNA breaks and mitochondrial dysfunction leading to activation of cell death pathways and accumulation of ROS. Early cardiac injury can be detected through quantification of circulating cTnl. The protective activity of DRZ against anthracycline cardiotoxicity is now thought to be largely mediated through inhibition of anthracycline binding to Top $2 \beta$. cTnl, cardiac troponin I; DRZ, dexrazoxane; LV, left ventricular; ROS, reactive oxygen species. of stay and overall mortality. In a study of over 11000 consecutive patients undergoing coronary artery bypass graft (CABG) between 2002 and 2010, postoperative AF occurred in $29.5 \%$ of patients with no previous AF diagnosis, with no change in incidence over this time period (figure 2). ${ }^{3}$ The adjusted median time from surgery to AF onset was 52 hours with a median total time in AF of 22 hours per patient.

The pathophysiology of AF after CABG surgery has not been fully elucidated and cannot be explained by surgical trauma of atrial tissue given that cardiopulmonary bypass for CABG surgery typically does not include significant manipulation of the left atrium. Thus, as Akoum ${ }^{4}$ suggests in an editorial, it is likely "that post-CABG AF shares common mechanisms with AF that is not associated with surgery. These include the firing of electrical triggers, a vulnerable atrial substrate composed of both electrical and structural tissue remodelling, all in the presence of a proarrhythmic autonomic milieu." Many of these patients also have clinical risk factors for AF, so that "The likely cause of this stable incidence is that these risk factors are associated with the development of atrial fibrosis which creates a substrate for AF." Whether or not we can prevent post-CABG AF by risk factor modification or targeted medical therapy remains an open question; clearly our treatments to date have been ineffective from a population perspective.

Also in this issue of Heart is a stateof-the-art review article on anthracycline cardiotoxicity by Hendricksen ${ }^{5}$ which discusses mechanism of cardiomyocyte injury, as well as recommendations for monitoring and prevention (figure 3 ).

The Education in Heart article discusses the causes of sudden death and recommendations for ECG screening for heart disease in athletes. ${ }^{6}$ You can listen to a short summary and discussion of this article in a podcast with James Rudd interviewing Dr Andrew D'Silva from Brighton, UK, one of the authors of the article. https://soundcloud.com/bmjpodcasts/ecgscreening-for-young-athletes-yes-or-no? in =bmjpodcasts/sets/heart-podcast

The Image Challenge in this issue ${ }^{7}$ provides a quick review of the causes of left ventricular outflow obstruction and how to differentiate each on echocardiographic imaging.

Competing interests None declared.

Patient consent Not required.

Provenance and peer review Commissioned; internally peer reviewed. 
(c) Article author(s) (or their employer(s) unless otherwise stated in the text of the article) 2018. All rights reserved. No commercial use is permitted unless otherwise expressly granted.

A Check for updates

To cite Otto CM. Heart 2018;104:961-963.

Heart 2018;104:961-963.

doi:10.1136/heartjnl-2018-313460

\section{REFERENCES}

1 Witte KK, Patel PA, Walker AMN, et al. Socioeconomic deprivation and mode-specific outcomes in patients with chronic heart failure. Heart 2018;104:993-8.

2 Squire I. Socioeconomic status and outcomes in heart failure with reduced ejection fraction. Heart 2018;104:966-7.

3 Filardo G, Damiano RJ, Ailawadi G, et al. Epidemiology of new-onset atrial fibrillation following coronary artery bypass graft surgery. Heart 2018;104:985-92.
4 Akoum N. Persistently high incidence of postcoronary bypass atrial fibrillation: possible mechanisms. Heart 2018;104:964-5.

5 Henriksen PA. Anthracycline cardiotoxicity: an update on mechanisms, monitoring and prevention. Heart 2018;104:971-7.

6 Lampert R. ECG screening in athletes: differing views from two sides of the Atlantic. Heart 2018;104:1036-42.

7 Spina R, lyer A, Jansz P. 56-year-old lady with transaortic gradient. Heart 2018;104:1043-4. 